\title{
Experimental analysis of the dynamic inflow effect due to coherent gusts
}

\author{
Frederik Berger ${ }^{1}$, Lars Neuhaus ${ }^{1}$, David Onnen ${ }^{1}$, Michael Hölling ${ }^{1}$, Gerard Schepers ${ }^{2,3}$, and \\ Martin Kühn ${ }^{1}$ \\ ${ }^{1}$ ForWind - Center for Wind Energy Research, University of Oldenburg, Institute of Physics, Küpkersweg 70, 26127 \\ Oldenburg, Germany \\ ${ }^{2}$ TNO Energy Transition, Petten,1755 LE, The Netherlands \\ ${ }^{3}$ Hanze University of Applied Sciences, Institute of Engineering, Groningen, 9747 AS, the Netherlands
}

Correspondence: Frederik Berger (frederik.thore.berger@uol.de)

\begin{abstract}
The dynamic inflow effect describes the unsteady aerodynamic response to fast changes in rotor loading, due to the inertia of the wake. For pitch actuation and fast rotor speed changes this effect leads to load overshoots. The effect is suspected to be also relevant for gust situations, however this was never shown. The objective of the paper is to proove the dynamic inflow effect due to gusts and compare dynamic inflow engineering models to corresponding measurements. A $1.8 \mathrm{~m}$ diameter model turbine is used in the large wind tunnel of ForWind - University of Oldenburg with an active grid to impress rotor uniform gusts on the flow. The campaign features load and velocity measurements of the axial flow in the rotor plane. The unsteady dynamic inflow effect is investigated by comparing two experimental cases. Firstly, a dynamic measurement during a gust situation is performed. Secondly, quasi-steady loads and axial velocities are interpolated from a steady characterisation experiment according to the gust wind speed. By comparing both cases, the influence attributed to the dynamic inflow effect is isolated. Further comparisons to a typical Blade Element Momentum code and a higher fidelity Free Vortex Wake Model are performed. Based on analytical considerations an improvemed formulation of the Øye dynamic inflow model is proposed. The experiment shows a dynamic inflow effect due to gusts in the loads and axial velocity measurements. It leads to a reduction in load and axial velocity amplitudes and consequently also lower fatigue loading. The higher fidelity model shows a similar impact of the dynamic inflow effect. In contrast, the commonly used Øye engineering model in the BEM code predicts an increase in load amplitude and thus higher fatigue loads. The improved Øye engineering model however catches the observed dynamic inflow effect due to gusts in accordance to the experiment and FVWM simulations. An amplification of induced velocities, seen in the experiment and FVWM simulation, causes the reduced load amplitudes. Therefore, classic dynamic inflow models, which filter the induced velocity, cannot predict the effect. The proposed improvement to additionally consider the wake velocity for the filter of the dynamic inflow engineering model, proves to be a straight forward but also effective modification. In conclusion, these new experimental findings on dynamic inflow due to gusts and improvements to the Øye model enable improvements in wind turbine design by catching the related lower fatigue loads.
\end{abstract}




\section{Introduction}

The dynamic inflow phenomenon is an unsteady aerodynamic effect relevant for helicopters (Peters, 2009) and wind turbines (Snel and Schepers, 1994). It is considered for a fast load variation through a blade pitch step or fast change in rotor speed. Due to the inertia of the wake, the induced velocity cannot change instantanously but only gradually to a fast load event in the rotor plane. This dynamic transition in the wake leads to load and power overshoots between the two steady states. For wind turbines, Snel and Schepers (1994) also suspect variations in inflow velocity, especially due to coherent wind gusts, to lead to relevant dynamic inflow effects.

Aeroservoelastic simulations are used to obtain relevant turbine loads in the design and certification process of wind turbines.

The aerodynamic part of these simulations is based on the Blade Element Momentum (BEM) theory, that gives the aerodynamic forces acting on the rotor blade segments. BEM however is inherently based on steady flow assumptions and engineering models are needed to catch dynamic phenomena, like the dynamic inflow effect. Widely used examples are the ECN model (Snel and Schepers, 1994) in Phatas and the ECN (now TNO) Aero-Module, the recent DTU model (Madsen et al., 2020) in HAWC2 and the Øye model (Snel and Schepers, 1994) in GH Bladed and OpenFAST. They all have the main working mechanism that they filter the induced velocitiy based on time constants. One time constant is used for the ECN model and two time constants for the DTU and Øye model.

Recently Perez-Becker et al. (2020) and Boorsma et al. (2020) compared aeroelastic simulations based on Blade Element Momentum (BEM) theory and Free Vortex Wake Methods (FVWM). In addition to the need of engineering models for dynamic inflow effects, BEM is based on the assumption of axial and uniform inflow. Current BEM tools used in industry and academia have different implementations to handle non-uniform inflow (Boorsma et al., 2020). FVWM on the other hand models dynamic inflow effects and non-uniform inflow intrinsically. Both investigations looked at the influence of turbulent wind fields with shear on the loading of wind turbines. Both found relevant lower fatigue loading for the out-of-plane blade root bending moments and tower bottom fore-aft bending moment for the higher fidelity FVWM type simulations. The implementations of non-uniform inflow in BEM was identified as one main contribution to this behaviour in both investigations. Boorsma et al. (2020) also suspected the dynamic inflow effect to be responsible for some of the differences between BEM and FVWM in turbulent inflow.

Within two historic EU projects on dynamic inflow (Snel and Schepers, 1994; Schepers and Snel, 1995) a 1.2 m diameter model wind turbine was exposed to a step change in wind velocity generated by a manually operated gust generator in a wind tunnel. This experiment did not give a clear indication for a dynamic inflow effect due to gusts. Snel and Schepers (1994) related this to the slow step change in wind velocity, compared to the typical dynamic inflow time constant. Simulations with engineering models suggested a slight load overshoot for the investigated case. Shirzadeh et al. (2021) investigated dynamic load and power characteristics due to extreme shear and gusts based on tower base force and power measurements. They used a $2.2 \mathrm{~m}$ diameter model wind turbine with very low design tip speed ratio and constant generator resistance in the WindEEE Dome. They exposed it to a gust, where the wind velocity increased from $5 \mathrm{~ms}^{-1}$ to $9 \mathrm{~ms}^{-1}$ and back again within $5 \mathrm{~s}$. They neither looked specifically into the dynamic inflow phenomenon, nor is an effect clearly indicated in the presented plots. 
Until now, there is no conclusive information on the relevance or even existence of dynamic inflow effects due to gusts. Consequently, it is also not known if current engineering models can model this expected effect.

The objective of this work is to experimentally proove and quantify the dynamic inflow effect due to gusts and to investigate the behaviour in engineering models. The work continues and builds on the methods of the radially resolved induction factor measurements of a pitch step experiment in Berger et al. (2021a) and the comparison of that experiment to engineering models in Berger et al. (2020), however here for gusts. An active grid is used in the wind tunnel to create coherent gust situations. Two experimental cases are compared to extract the dynamic inflow effect due to a gust, as the difference between those cases. The first case is a dynamic measurement of loads and axial velocity in the rotor plane. Secondly, a quasi-steady measurement is emulated by interpolation from a detailed experimental characterisation of loads and axial velocity, based on the gust velocity. An enhancement for gusts is proposed based on analytical assumptions for engineering models and implemented for the Øye dynamic inflow model. The dynamic inflow behaviour due to gusts of the original and the gust-improved Øye dynamic inflow model and a FVWM are compared to the experimental behaviour.

\section{Methods}

Within this study various methods are combined and a short overview of the methods is given as a guidline. Firstly the experimental setup is introduced (Sect. 2.1). In the following the gusts are quantified (Sect. 2.2). In the measurement matrix all measurement cases, positions and repetitions are outlined (Sect. 2.3). Moving forward, the wake induction measurements and the additional load reconstruction from the flow measurements is introduced (Sect. 2.4). The construction of the dynamic (Sect. 2.5) and the quasi-steady (Sect. 2.6) case is described in the next step. Following, the concept of dynamic inflow engineering models is outlined and an enhancement for gust situations proposed (Sect. 2.7). In the last part the simulation models for BEM and FVWM are presented (Sect. 2.8).

\subsection{Experimental setup}

\section{Wind tunnel}

The experiments were performed in the large wind tunnel of ForWind - University of Oldenburg. The Göttingen type wind tunnel has an outlet section of $3 \mathrm{~m}$ by $3 \mathrm{~m}$. The wind tunnel was operated in an open jet test section configuration with a test section length of $30 \mathrm{~m}$ (maximum wind velocity of $32 \mathrm{~ms}^{-1}$ ). The wind tunnel is described in more detail in Kröger et al. (2018).

\section{Active grid}

An active grid is attached to the wind tunnel nozzle to manipulate the flow (Kröger et al., 2018; Neuhaus et al., 2021). The grid consists of 80 individually controllable shafts with rectangular flaps to control the distribution of blockage of the flow. Thus wind variations can be repeatedly generated, as shown by Kröger et al. (2018). The active grid is shown in Fig. 1 a. 

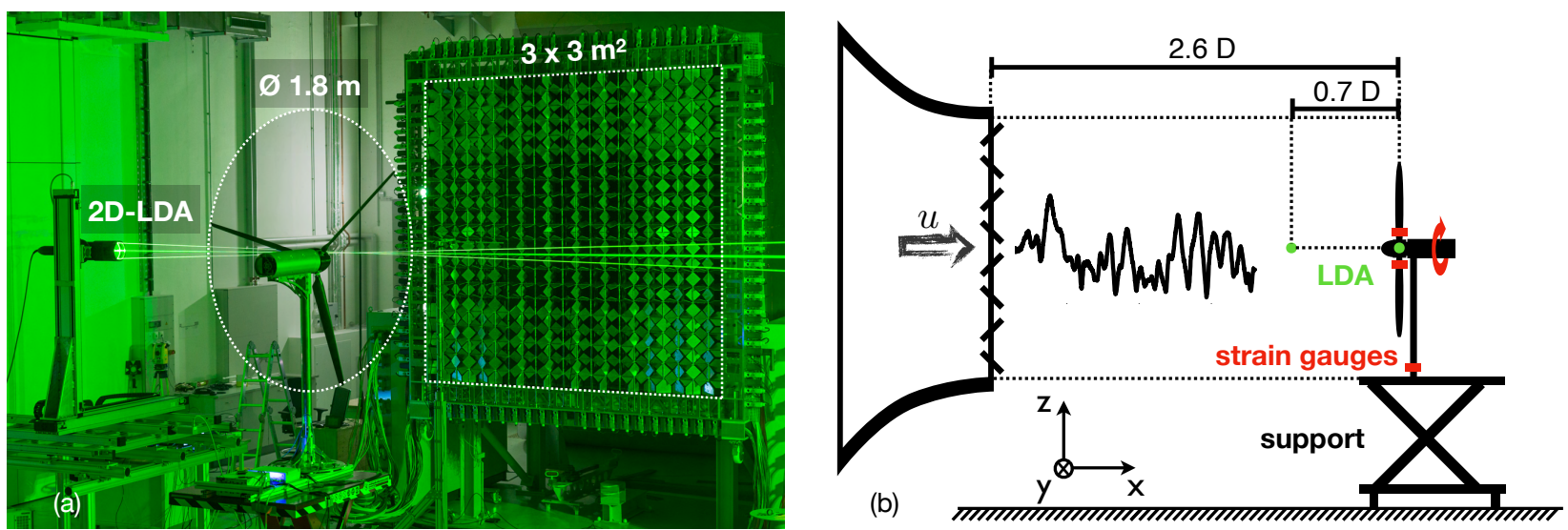

Figure 1. (a) Picture of MoWiTO 1.8 and 2D-LDA in wind tunnel with active grid. Note the visible shift of grid flap angles between the centered inner square and outer compensating axes, demonstrated in the picture for a smaller inner square part than used in the investigation. (b) Schematic of setup with coordinate system in the wind tunnel.

\section{Model turbine}

The Model Wind Turbine Oldenburg with a diameter $(D)$ of $1.8 \mathrm{~m}$ (MoWiTO 1.8) is used for the investigation (see Fig. 1a). The turbine is aerodynamically scaled based on the NREL 5 MW reference turbine (Jonkman et al., 2009) and maintains the design TSR, thrust and power characteristics, as well as the non-dimensional lift and thus induction distribution. The turbine blades are scaled by a geometrical factor of $n_{\text {length }}=\frac{1}{70}$. Influenced by structural constraints the time scaling of the design is $n_{\text {time }}=50$. This leads to $n_{\text {time }}$-times faster rotor speeds, $\frac{1}{n_{\text {time }}}$ gust length, as well as $\frac{n_{\text {time }}}{n_{\text {length }}}$ factor on the wind velocity. Low Reynolds number profiles are used for the stiff carbon fibre made blades. The blades have an estimated maximum tip deflections of less than $0.015 \mathrm{~m}$ at the maximum wind velocity $\left(8.6 \mathrm{~ms}^{-1}\right)$ in this study and a first eigenfrequency of $32 \mathrm{~Hz}$. The turbine has no rotor tilt or blade coning. It features individual pitch motors, torque control, encoders for rotor rotation and position. Further, strain gauges for flapwise blade root bending moment, rotor torque and tower bottom bending moment (to derive the rotor thrust) are used. The turbine position in the wind tunnel is shown in Fig. 1 b. Along the distance of $2.6 D$ behind the wind tunnel nozzle the induction zone of the turbine can freely develop (see Medici et al. (2011)). The turbine control and data acquisition is handled by a National Instruments Compact Rio system. The turbine scaling and design are described in detail in Berger et al. (2018).

\section{Laser Doppler Anemometer}

A 2D Laser Doppler Anemometer (LDA) by TSI Inc., with a beam expander with a focus length of $2.1 \mathrm{~m}$, is used. The optical measurement device is placed well outside of the wind flow. It is mounted on a motor driven three directional traverse with $1.5 \mathrm{~m}$ travel distance in each direction (see Fig. 1a). A LDA has no fixed sampling frequency but depends on various parameters, including the seeding of the wind flow with small oil particles. Typical LDA sampling frequencies in this experiment are $2 \mathrm{kHz}$. 


\subsection{Wind fields}

In this research the active grid is controlled by an overall constant blockage approach, where the inner square part $(2 \mathrm{~m}$ by $2 \mathrm{~m})$ of the grid impresses the desired gust conditions. The outer axes are used to keep the mean blockage of the grid constant. This way, fast velocity fluctuations with a high amplitude and degree of coherence can be achieved (Neuhaus et al., 2021). Two different types of transient wind fields with a high coherence over the swept area of the turbine are designed for this study. The wind tunnel fans are operated at constant speed and the velocity patterns are impressed by the flaps of the active grid. The first wind field is based on a continuous sinusoidal wind velocity variation (sine) with a frequency of $1 \mathrm{~Hz}$, mean wind velocity of $6.4 \mathrm{~ms}^{-1}$ and an amplitude of $1.8 \mathrm{~ms}^{-1}$. The second wind field has effectively $20 \mathrm{~s}$ of stochastic variations (stochastic), with a mean wind velocity of $6.2 \mathrm{~ms}^{-1}$, maximum value of $8.6 \mathrm{~ms}^{-1}$ and minimum value of $4.4 \mathrm{~ms}^{-1}$.

The wind fields are quantified based on LDA measurements at 15 positions $0.7 \mathrm{D}$ upstream of the turbine at standstill. The streamwise position of the measurements is indicated in Fig. $1 \mathrm{~b}$. The positions are located in a y-z plane (parallel to rotor plane). We measure on a horizontal (-y direction) and diagonal line (-y,-z direction) starting from the centerline of the wind tunnel in steps of $0.2 R$ to $0.8 R$ and finer step width of $0.1 R$ from $0.8 R$ to $1.1 R$, where $R$ is half of the rotor diameter $D$. Due to the symmetry of the square grid the measurement points within the considered quarter of the rotor give a good representation of the whole swept area. For the sine variation 40 repetitions are considered and for the stochastic variation 10 per position.

These measurements are synchronised with the grid movement. For each wind field, a spatial mean wind velocity is defined by firstly binning all time series of the positions up to $0.9 R$ from the rotor axis to bins of $0.01 \mathrm{~s}$ and then averaging each bin. This gives a $100 \mathrm{~Hz}$ sampled spatial mean wind signal, as shown in Fig. 2 a, b.

The level of uniformity over the swept area is assessed based on the Pearson correlation coefficient, as a measure of correlation of the wind velocity at single positions to the mean wind signal. It is calculated for the separately binned and averaged different positions in comparison to the spatial mean wind signal. These values are plotted colour coded at the measurement positions alongside relevant turbine dimensions in Fig. $2 \mathrm{c}$, d. Additionally, a local stream-tube radius $\left(R_{e f f}\right)$ is plotted that takes into account the widening of the stream-tube due to the induction zone at a rotor averaged axial induction of $a=0.15$ for the running turbine.

Correlation coefficients within the local stream-tube radius are very high for both wind fields and for the sine variation for all measured positions, as expected based on the investigation by Neuhaus et al. (2021). For the stochastic variation the correlation decreases towards the edge of the active grid inner square, with still high values within $R_{e f f}$. The level of uniformity over the rotor swept area is evaluated as sufficient for the planned investigation. In the latter analyses, $95 \%$ Confidence Intervals (CI) are determined for various measured quantities. They are based on the binned data of all measurements within $R_{e f f}$.

Cross-correlation between the mean velocity $0.7 D$ upstream of the turbine and the spatial mean velocity of three standstill (locked rotor) measurements in the rotor plane $(0.4 R, 0.6 R$ and $0.8 R)$ is used to obtain the time delay the wind field needs to reach the turbine from the upstream characterisation point. This time delay is needed later to align the dynamic and quasi-steady measurement signals. Comparing the measurements $0.7 \mathrm{D}$ upstream of the turbine and in the rotor plane we only see minor differences between signals. To account for possible small changes in the wind field while travelling from $0.7 D$ upstream of 

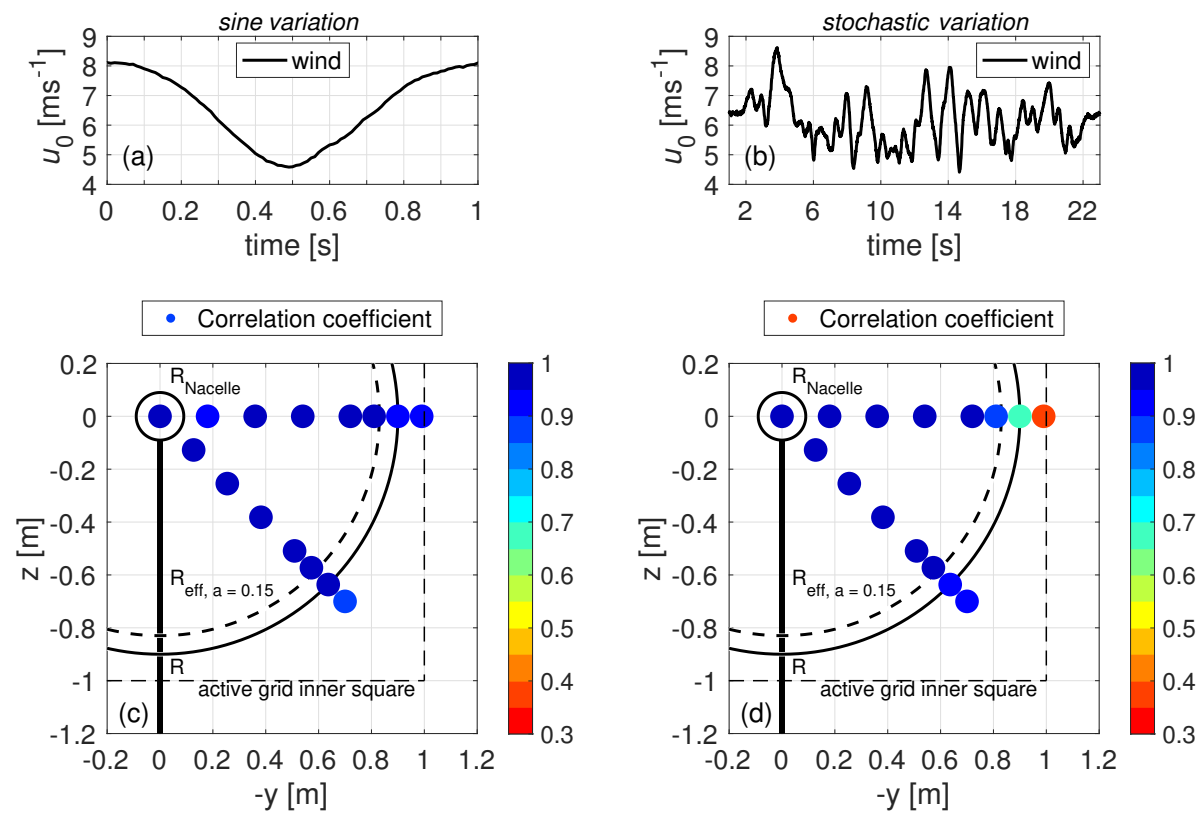

Figure 2. Undisturbed mean wind field of the sine (a) and stochastic (b) variation. Front view on the lower right hand side quarter of the swept area of the turbine looking downstream. The colour coded measurement positions show the Pearson correlation coefficient of the local measurement position wind velocity to the mean wind velocity for the sine (c) and stochastic (d) variation.

the turbine to the rotor plane the uncertainty band will be altered in latter analyses at few single instances, in order to always encase the spatial mean velocity measured in the rotor plane.

\subsection{Measurement matrix}

Aerodynamic rotor torque, thrust and flapwise blade root bending moments are obtained based on strain gauge measurements.

Additionally to the two wind fields, a staircase variation with 12 steps with a length of $25 \mathrm{~s}$ each and velocity range from $4.6 \mathrm{~ms}^{-1}$ to $9.0 \mathrm{~ms}^{-1}$ is performed for a turbine characterisation. For this wind field the speed of the wind tunnel fans is changed and the grid flaps are at the constant open position, acting as a passive grid. The turbine is operated at a steady rotational speed of $480 \mathrm{~min}^{-1}$ and a constant pitch setting of $1^{\circ}$ (towards feather) for all measurements with operating turbine.

The staircase wind field is characterised at five horizontal positions between $0.1 R$ and $0.9 R$ at $0.7 D$ upstream of the turbine. LDA measurements of the streamwise velocity are performed in the rotor plane at nine radial (along $\mathrm{x}$-axis) positions (for the range $0.3 R$ to $0.9 R$ in steps of $0.1 R$ and additionally at $0.25 R$ and $0.95 R$ ) for the operational sine and staircase variation. An overview of the test matrix with additional information on the repetitions for each wind field is given in Tab. 1. 
Table 1. Experimental test cases including number of measurement positions of the LDA and repetitions.

\begin{tabular}{|c|c|c|c|}
\hline \multirow[t]{2}{*}{ Turbine state } & \multicolumn{3}{|c|}{ Wind } \\
\hline & sine & stochastic & staircase \\
\hline \multirow[t]{3}{*}{ standstill } & LDA $0.7 \mathrm{D} / 0 \mathrm{D}$ & LDA $0.7 D / 0 D$ & LDA $0.7 D$ \\
\hline & 15 pos. / 3 pos. & 15 pos. / 3 pos. & 5 pos. \\
\hline & 40 rep. per pos. & 10 rep. per pos. & 1 rep. per pos. \\
\hline \multirow[t]{3}{*}{ operation } & LDA $0 D$ & no LDA & LDA $0 D$ \\
\hline & 9 pos. & - & 9 pos. \\
\hline & 40 rep. per pos. & 200 rep. & 1 rep. per pos. \\
\hline
\end{tabular}

pos. $=$ position; rep. $=$ repetition

\subsection{Wake inductions}

\section{Measurement}

The method by Herráez et al. (2018) is used to derive the wake induction factors. The local velocity in the rotor plane is used for the method and probed in the bisectrix of two blades. For axial and uniform flow the probed velocity is free of the influence of the bound circulation as the blade induction is counterbalanced and thus cancels out. Therefore the local velocity equals the radially averaged velocity in the rotor plane. For this axial and uniform flow the wake induction factors thus are equal to the induction factors. The method was developed for steady operation, however, it was shown in a prior study in Berger et al. (2021a) to be also applicable to study the transient changes in induction factors, maintaining axial and uniform conditions.

In Fig. 3 a the turbine is shown with the LDA laser beams and the probed axial $\left(u_{a x}\right)$ and tangential $\left(u_{t a}\right)$ velocity components at a specific radius. In Fig. $3 \mathrm{~b}$ the concept of the counterbalanced bound circulation of the evenly loaded blades is sketched. At the indicated line of measurement the downwash of the blade ahead of the indicated line counteracts the upwash of the blade behind it and they cancel each other. The blade at the 9'o clock position has no influence on the measurement. Herráez et al. (2018) outline that the trailed vorticity cannot be captured well due to the high distance between the measurement position and the blade tip. Therefore this method is less suited for the root and tip region of the blade. The results at $0.25 R$ and $0.95 R$ should therefore be interpreted with care. The application of this method to MoWiTO with the same 2D LDA setup is introduced and discussed in more detail in Berger et al. (2021a) (Sect. 2.1.3 and App. A.) The same threshold value for the bisectrix position of the rotor azimuth angle of $\pm 3^{\circ}$ was applied, as this showed to be a good compromise between data samples and quality.

The axial and tangential induction factors are defined by Eq. (1) and Eq. (2), respectively. The undisturbed inflow velocity is $u_{0}$ and the rotor angular velocity $\Omega$. With the geometrical angle of the blade segment $\gamma$, consisting of twist and pitch, the local angle of attack $\alpha$ ar radius $r$ can be calculated by Eq. (3). 


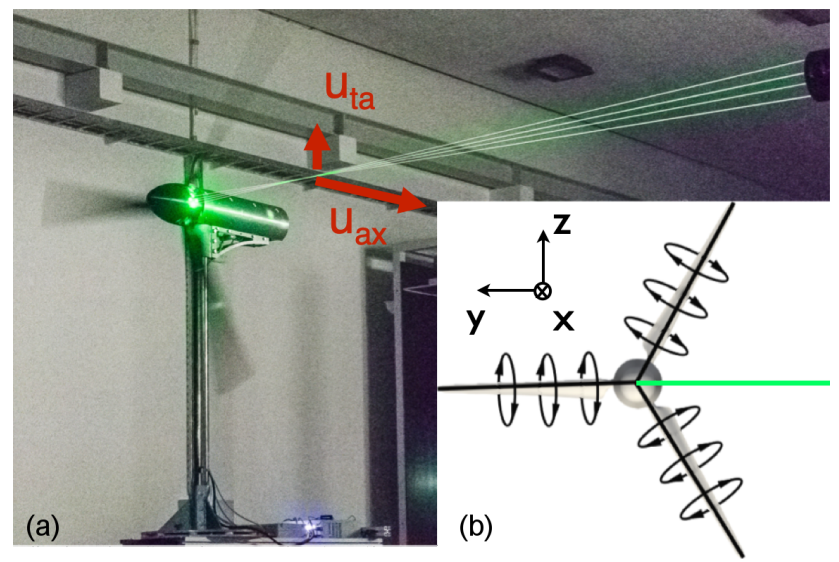

Figure 3. (a) MoWiTO 1.8 with 2D-LDA measurement in the bisectrix of two blades of axial and tangential velocity. (b) Counterbalancing of bound circulation for the evenly loaded blades (modified from Herráez et al. (2018)).

$a=1-\frac{u_{a x}}{u_{0}}$

$a^{\prime}=\frac{u_{t a}}{\Omega \cdot r}$

$\alpha=\arctan \left(\frac{u_{a x}}{u_{t a}+\Omega \cdot r}\right)-\gamma$

\section{Load reconstruction from inductions}

The measurements of $u_{a x}$ and $u_{t a}$ are further used to reconstruct the turbine loads. The approach is based on the blade element theory (BET), usually used within a BEM code as outlined in detail by Hansen (2008) to obtain the aerodynamic forces from the flow information. The relative velocity at the blade segments is defined by Eq. (4). The angle of attack along the span is derived from the experiment through Eq. (3). The aerodynamic forces for single blade elements are calculated in normal direction $F_{N}$ by Eq. (7) and in tangential direction $\left(F_{T}\right)$ by Eq. (8). The inflow angle is the sum of $\alpha$ and $\gamma$ and defined by $\theta$. The lift and drag forces of the segment are given by Eq. (5) and Eq. (6), respectively.

$$
\begin{aligned}
u_{r e l} & =\sqrt{u_{a x}^{2}+\left(u_{t a}+\Omega r\right)^{2}} \\
F_{L} & =\frac{1}{2} \cdot C_{L}(\alpha) \cdot \rho \cdot w \cdot c \cdot u_{r e l}^{2} \cdot F \\
F_{D} & =\frac{1}{2} \cdot C_{D}(\alpha) \cdot \rho \cdot w \cdot c \cdot u_{r e l}^{2} \cdot F \\
F_{N} & =F_{L} \cos \theta+F_{D} \sin \theta \\
F_{T} & =F_{L} \sin \theta-F_{D} \cos \theta
\end{aligned}
$$


The lift and drag coefficients are given by $C_{L}(\alpha)$ and $C_{D}(\alpha)$,respectively, and obtained from XFoil (Drela, 1989) simulations for the respective Reynolds numbers. 3D effects are accounted for through the correction model for the lift coefficient at high angles of attack by Snel et al. (1993), which is mainly relevant for the root sections. The width of a blade segment is defined by $\Delta r$ and the chord length by $c$. The tip losses are accounted for by the tip loss model by Shen et al. (2005) with the factor $F$. The segmented aerodynamic loads are integrated along the blade span to obtain the integral load signals.

\subsection{Dynamic experiment}

\section{Ensemble averaging and error estimation}

Ensemble averages are calculated for each of the loads from the various repetitions of the two dynamic cases. For the sine gust the ensemble average of the flapwise blade root bending moment $\left(M_{\mathrm{flap}}\right)$ is thus based on the $N=360$ repetions of the sine movement, according to Eq. (9).

$M_{\text {flap }}(t)=\frac{1}{N} \sum_{n=1}^{N} M_{\text {flap, single cycle, all blades }}^{(n)}(t)$

With this approach, noise and non-deterministic variations can be reduced. Even some deterministic fluctuations, like the blade-tower interaction is smoothed out as the start of the active grid wind protocols and the rotor azimuth position are not synchronised.

The LDA based induction measurement data for the sine variation are processed with a similar approach. The data points within the threshold in the bisectrix of the single repetitions are synchronised with the wind field and combined to one single signal. As the rotational frequency of $8 \mathrm{~Hz}$ of the rotor is a multiple of the frequency of the sine at $1 \mathrm{~Hz}$ we see 24 data point clusters for this three bladed turbine over one sine period. This data is binned to clusters and the mean value of each bin is taken as a representative value.

\section{Signal corrections}

Corrections are applied to the torque and thrust signals based on the strain gauge measurements. To obtain the aerodynamic rotor torque, the measurement signal is corrected for two effects. Firstly, the friction in the main shaft bearings and slipring is added to the measured torque. This correction increases the torque by $3 \%$ at the mean velocity of the wind fields. Secondly, there is an inertial effect of the rotor due to slight changes in the rotor speed up to $\pm 3 \%$, as the controller cannot keep the rotor perfectly constant for the fast changes in wind velocity. Equation (10) is used to correct the torque signal by the contribution $\Delta M(t)$ associated with the angular acceleration of the rotor $\dot{\Omega}$ and inertia of the rotor and drivetrain $I_{\text {rot }}$.

$\Delta M(t)=I_{\text {rot }} \cdot \dot{\Omega}(t)$

As introduced, dynamic inflow effects can also be triggered by fast changes in rotor speed. The influence of the slight changes in rotor speed in the presented experiment are investigated with a BEM tool with the Øye dynamic inflow engineering model 
https://doi.org/10.5194/wes-2022-2

Preprint. Discussion started: 18 January 2022

(c) Author(s) 2022. CC BY 4.0 License.

(c) (1)

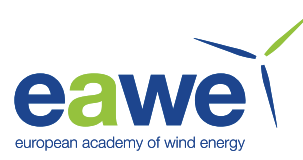

(see Sect. 2.7). As a result the influence of the dynamic inflow effect due to the rotor speed changes is considered negligible in the context of this work.

The thrust is derived from the tower bottom bending moment in fore-aft direction. The measurement is corrected for the influence of tower and nacelle drag. This drag was estimated based on a quadratic fit to a measurement of the tower bottom bending moment at various wind speeds with the turbine without installed blades.

\subsection{Quasi-steady behaviour}

The quasi-steady case is based on a detailed characterisation of the turbine with the staircase wind protocol. Turbine loads are represented in a non-dimensional form. The uncommon blade root bending moment coefficient $C_{\text {flap }}$ is defined in Eq. (11). The reference bending moment is the denominator of the thrust coefficient multiplied with an additional characteristic length of $\frac{2}{3} R$, based on the representative attack point of the load for an idealised triangular normal force distribution, and the reciprocal of the number of blades $i$. $C_{\text {flap }}$ and the common thrust coefficient $C_{\mathrm{T}}$ and torque moment coefficient $C_{\mathrm{M}}$ are presented over TSR in Fig. 4.

$C_{\text {flap }}=\frac{M_{\text {flap }}}{\frac{1}{i} \frac{\rho}{2} \rho u_{0}^{2} \pi R^{2} \frac{2}{3} R}$

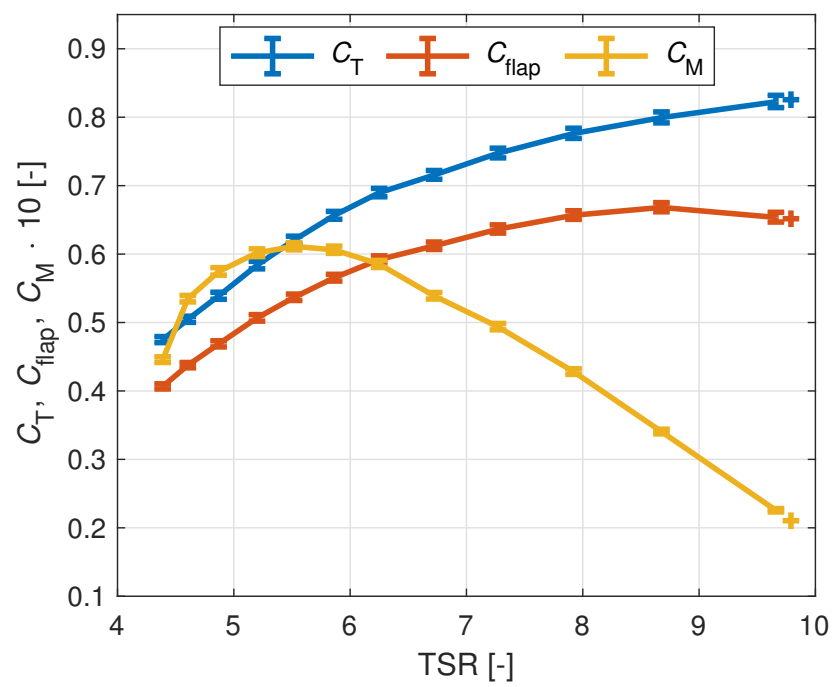

Figure 4. Turbine characteristics for construction of quasi-steady case. Relevant TSR range for the sine and stochastic wind variation is TSR 5.6 to 9.5 and 5.4 to 9.8 , respectively.

Errorbars indicate the quadratically added up uncertainty of inflow wind velocity and the $95 \%$ CI of the load measurement for the 20 s long considered measurement length per wind velocity step. The additional plus sign represents an extrapolated value slightly outside of the staircase wind protocol. This extra value is needed to construct the quasi steady loads at a single negative wind gust for the stochastic wind variation case. 
The axial (see Eq. (1)) and tangential (see Eq. (2)) induction factors are obtained with the same staircase wind protocol. Based on these also the angle of attack (see Eq. (3)) is obtained. Three representative distributions over the radius are shown for chosen TSR in Fig. 5 a, b and c.
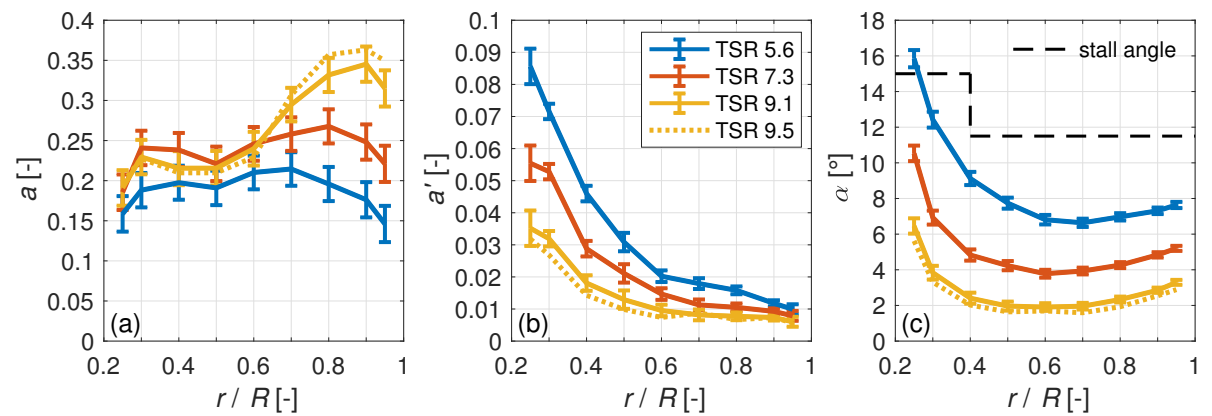

Figure 5. Distributions of (a) axial induction factor, (b) tangential induction factor and (c) angle of attack for different TSR. The dotted line represents a linear extrapolation.

The solid lines represent the highest, lowest and middle operational TSR configurations for the sine protocol within the characterisation. The errorbars indicate the quadratic error of the inflow uncertainty and the $95 \% \mathrm{CI}$ of the induction measurement for the inductions and the propagated error for the angle of attack. Another high TSR state is extrapolated and shown in dashed lines. This extraploation however is only minor. At the highest TSR operational point in the characterisation, the rotor speed dropped due to the chosen controller settings. This led to a lower TSR value than needed for the construction of the quasi-steady signals. For the load characterisation, additional characterisations at higher rotor speed were recorded. Therefore the highest recorded TSR in the characterisations of loads and inductions differ.

The trends for the axial and tangential induction and angle of attack are as expected. The axial induction factor (Fig. 5 a) for the low and middle TSR setting shows a unifom spanwise distribution. We see higher values of $a$ for higher TSR. For the high TSR settings these values in the inner rotor half decrease again, whereas the axial induction factor is increased in the outer rotor half, compared to the TSR 7.3 case. Axial induction factors are below the design induction factor for optimal power extraction of $1 / 3$, apart from the tip region at the high TSR settings where the maximum value is found at 0.36 . Thus the turbine is not operating within the turbulent wake state throughout the experiments, as can also be seen at the maximum $C_{\mathrm{T}}$ value of 0.83 .

The tangential induction factor (Fig. 5 b) has high values near the root, which decrease towards the outer part. Also, the tangential induction values generally decrease with increasing TSR for the respective radii, as expected from the decreasing momentum coefficient from the low to high TSR (see Fig. 4).

The angle of attack (Fig. $5 \mathrm{c}$ ) is higher towards the root and similar for the range from $0.5 R$ to the tip. The general distributions show the highest radius dependent angles of attack for the low TSR setting and decrease with increasing TSR. The stall angle is estimated based on the highest lift coefficient at $15^{\circ}$ for the root airfoil up to $0.4 R$ and at $11.5^{\circ}$ for the airfoil used from $0.5 R$ to the tip. This limit is exceeded at the lowest TSR setting for the radius at $0.25 R$. For the remaining radii at this TSR and higher TSR in general, the angles of attack are within the stall limits. 
The quasi-steady turbine loads and inductions, shy off dynamic inflow effects, are obtained for the dynamic wind field by interpolation from this characterisation. The reference wind speed of the gust at the rotor plane position is used for the construction. This reference speed is the mean of the measured 15 positions in front of the turbine and a time delay was obtained based on minimizing the root mean square error in the cross correlation of the mean wind to the mean velocity of the three reference wind measurements in the rotor plane.

\section{7 Øye dynamic inflow model and improved formulation for gusts}

In BEM simulations, engineering models are needed to catch the dynamic inflow effect. By filtering the induced velocity, the inertia of the wake is considered, leading to a load overshoot for a pitch step. For constant wind velocity $u_{0}$ the axial induction $a$ and far wake velocity $u_{2}$ show the same gradual change between two steady operational states as the induced wind velocity $u_{\text {ind }}$, as $u_{\text {ind }}=a \cdot u_{0}$ and $u_{2}=u_{0}(1-2 a)$, respectively. Consequently applying the filter on $a$ or $u_{2}$ would not change the behaviour of the model, given constant $u_{0}$.

The dynamic inflow effect due to a pitch step should be described by two time constants (Pirrung and Madsen, 2018; Yu et al., 2019; Berger et al., 2021a). The faster time constant $\tau_{\text {fast }}$ can be attributed to the sudden change in the trailed vorticity and the slower time constant $\tau_{\text {slow }}$ to the effect of the wake inertia.

A gust can also lead to fast changes in $u_{\text {ind }}$ within relevant time scales to the dynamic inflow effect $\left(\tau_{\text {typ }}=R / u_{0}\right.$, see Snel and Schepers, 1994). Such gust cases differ physically from the more classic dynamic inflow cases of a fast pitch step or rotor speed change. Considering a turbine model without any dynamic effects at constant rotational speed, a fast increase in $u_{0}$ due to a gust leads to a decrease in $a$ (due to the reduced TSR). With the induced velocity being $u_{\text {ind }}=a \cdot u_{0}$, the effect of increase in $u_{0}$ on $u_{\text {ind }}$ is partly compensated by the decrease of $a$. Therefore the dynamic inflow effect is expected to be less significant than for a pitch step (only changes $a$ ), as outlined in Snel and Schepers (1994).

Following are the formulations of the Øye dynamic inflow model and the improved implementation of the same model. The improved formulation is based on the assumption, that the unsteady effect of the inertia of the far wake during gusts would be better caught by applying the corresponding filter function with $\tau_{\text {slow }}$ on the wake velocity $u_{2}$, rather than $u_{\text {ind }}$.

\section{Øye model}

In the Øye dynamic inflow model the steady induced velocities are filtered through two first order differential equations as in Eq. (12, 13) (Snel and Schepers, 1994; Hansen, 2008).

$$
\begin{aligned}
u_{\text {ind }, \text { int }}+\tau_{\text {slow }} \frac{d u_{\text {ind,int }}}{d t} & =u_{\text {ind, qs }}+k \cdot \tau_{\text {slow }} \frac{d u_{\text {ind, qs }}}{d t} \\
u_{\text {ind }}+\tau_{\text {fast }} \frac{u_{\text {ind }}}{d t} & =u_{\text {ind,int }}
\end{aligned}
$$

$u_{\text {ind, qs }}$ is the quasi-steady induced velocity, $u_{\text {ind,int }}$ an intermediate and $u_{\text {ind }}$ the final filtered induced velocity. The time constants $\tau_{\text {slow }}$ and $\tau_{\text {fast }}$ are defined by Eq. (14) and Eq. (15), respectively and their weighting ratio $k$ by Eq. (16). 


$$
\begin{aligned}
\tau_{\text {slow }} & =\frac{1.1}{(1-1.3 a)} \frac{R}{u_{0}} \\
\tau_{\text {fast }} & =\left[0.39-0.26\left(\frac{r}{R}\right)^{2}\right] \tau_{\text {slow }} \\
k & =0.6
\end{aligned}
$$

\section{Improved formulation of Øye model for gusts}

The motivation is that the delay function with $\tau_{\text {slow }}$ is meant to model the inertia of the wake. Considering a fast step-like increase in wind and resulting increase in induced velocity, a delay on the induced velocity would result in an overshoot of the far wake velocity, which can be expressed as $u_{2}=u_{0}-2 \cdot u_{\text {ind }}$.

The improved formulation of the Øye model thus considers the filter function with $\tau_{\text {slow }}$ to act on the velocity in the far wake, instead of the induced velocity. With this approach unphysical velocity overshoots in the inert wake due to fast gusts are prevented. The filter function with $\tau_{\text {fast }}$, related to the fast change in trailed vorticity, is left unchanged. The improved formulation is given in Eq. (17, 18), whereas Eq. (14-16) remain unchanged.

$$
\begin{aligned}
u_{2, \text { int }}+\tau_{\text {slow }} \frac{d u_{2, \text { int }}}{d t} & =u_{2, q s}+k \cdot \tau_{\text {slow }} \frac{d u_{2, q s}}{d t} \\
u_{\text {ind }}+\tau_{\text {fast }} \frac{d u_{\text {ind }}}{d t} & =\frac{u_{o}-u_{2, \text { int }}}{2}=u_{\text {ind }, \text { int }}
\end{aligned}
$$

Instead of the induced velocity ( $\left.u_{\text {ind }}\right)$ the far wake velocity $\left(u_{2}\right)$ is filtered in the first step by the slow time constant $\tau_{\text {slow }}$ in Eq. (17). In the second step the induced velocity, is filtered by the fast time constant as before in Eq. (18). The right side of that equation is the intermediate induced velocity as before, just expressed based on the intermediate far wake velocity.

\subsection{Comparing simulations}

Two different kinds of simulations, a BEM and a FVWM based, are used for comparison with the experimental data. For the BEM simulation the dynamic inflow engineering model can be disabled. Quasi-steady cases are generated for the FVWM simulation as for the experiment by characterisation with a staircase wind input in the respective simulation setup. The same airfoil polars as in Sect. 2.4 are used.

The first simulation environment is a BEM model programmed in MatLab and based on Hansen (2008). The BEM program considers axial and uniform inflow, equal loading for all blades and features a Prandtl tip loss model and high thrust correction (Buhl, 2005). The Øye dynamic inflow engineering model and the improved version for gusts (see Sect. 2.7) are implemented.

The second simulation environment is the FVWM model implemented in QBlade (Marten et al., 2016). It is based on the principles of Van Garrel (2003). The flowfield is modelled as a potential flow. The MoWiTO blade is discretised in 15 elements, which are each modelled by a bound ring vortex, thus forming a lifting line. The circulation of these vortices is calculated iteratively based on the airfoil polars and relative velocity. The vorticity is shed and trailed at each time step. The wake convection is obtained by forward integration with a first-order method. The induced velocity is influenced by the physical 
representation of the convecting wake, thus intrinsically modelling the dynamic inflow effect. The wake of twelve revolutions is considered and the azimuthal discretisation is $10^{\circ}$.

Both model setups were already used in Berger et al. (2020) and showed a good match to an experimental dynamic inflow focused pitch step experiment (Berger et al., 2021a) with MoWiTO. For both models neither unsteady profile aerodynamics nor structural flexibility are considered, but only the aerodynamic degrees of freedom at constant rotation.

\section{Results}

At first, the integral loads for sine and stochastic wind fields are presented, comparing the quasi-steady and dynamic experiment. Following, the radius resolved axial velocity and induced velocity of the sine gust is investigated. The thrust force is reconstructed based on these induction measurements. Lastly, a comparison of thrust and induced velocity for the sine gust of BEM and FVWM simulations to the experiment is presented.

\subsection{Loads}

\section{Sine inflow variation}

In Fig. 6 the sine wind field (a) and loads for flapwise blade root bending moment $M_{\text {flap }}$ (b), thrust $F_{\text {thrust }}$ (c) and aerodynamic rotor torque $M_{\text {aero }}$ (d) of quasi-steady and experiment case are plotted over time. The uncertainty band around the wind field shows the $95 \%$ CI of the sine wind field altered to higher error at few positions to enclose the mean wind vector based on the three standstill measurements in the rotor plane (see Sect. 2.2). These alterations are mainly in the range $t=0.1 \mathrm{~s}$ to $t=0.2 \mathrm{~s}$ and $t=0.6 \mathrm{~s}$ to $t=0.7 \mathrm{~s}$. The uncertainty band around the quasi-steady loads accounts for the quadratically added uncertainty in the wind velocity and the estimated error in the load characterisation (see Fig. 4). The uncertainty of the dynamic experiment shows the $95 \% \mathrm{CI}$ of the load measurement.

In the comparison of the quasi-steady case with the experiment case, the three considered load channels show similar behaviour. At the positive gust peak at $t=0 \mathrm{~s}$ steady and experiment case show similar values, but they differ at the negative gust peak at $t=0.5 \mathrm{~s}$. Here the experiment shows higher absolute loads than the quasi-steady case, thus leading to a reduction in load amplitude of the dynamic experiment by $20 \%$ to $23 \%$, based on the amplitude of the quasi-steady case.

The steady and dynamic curves differ mainly for the range of $t=0.3 \mathrm{~s}$ to $t=0.6 \mathrm{~s}$, where the wind velocity decreases quickly and the turbine operates at high TSR and thus thrust coefficients. The dynamic loads react with a reduced change in load from $t=0.3 \mathrm{~s}$ on and reach the quasi-steady curve again after the wind velocity increases at $t=0.6 \mathrm{~s}$. For the torque, this behaviour can also be seen less pronounced for the increasing wind velocity slope around $t=0.8 \mathrm{~s}$.

\section{Stochastic inflow variation}

Figure 7 shows the wind velocity of the stochastic wind variation in (a), the difference quotient of the rotor equivalent induced velocity to a relevant dynamic inflow time constant $\tau$ in (b) and $M_{\text {aero }}$ for the quasi-steady and dynamic case in (c). The 

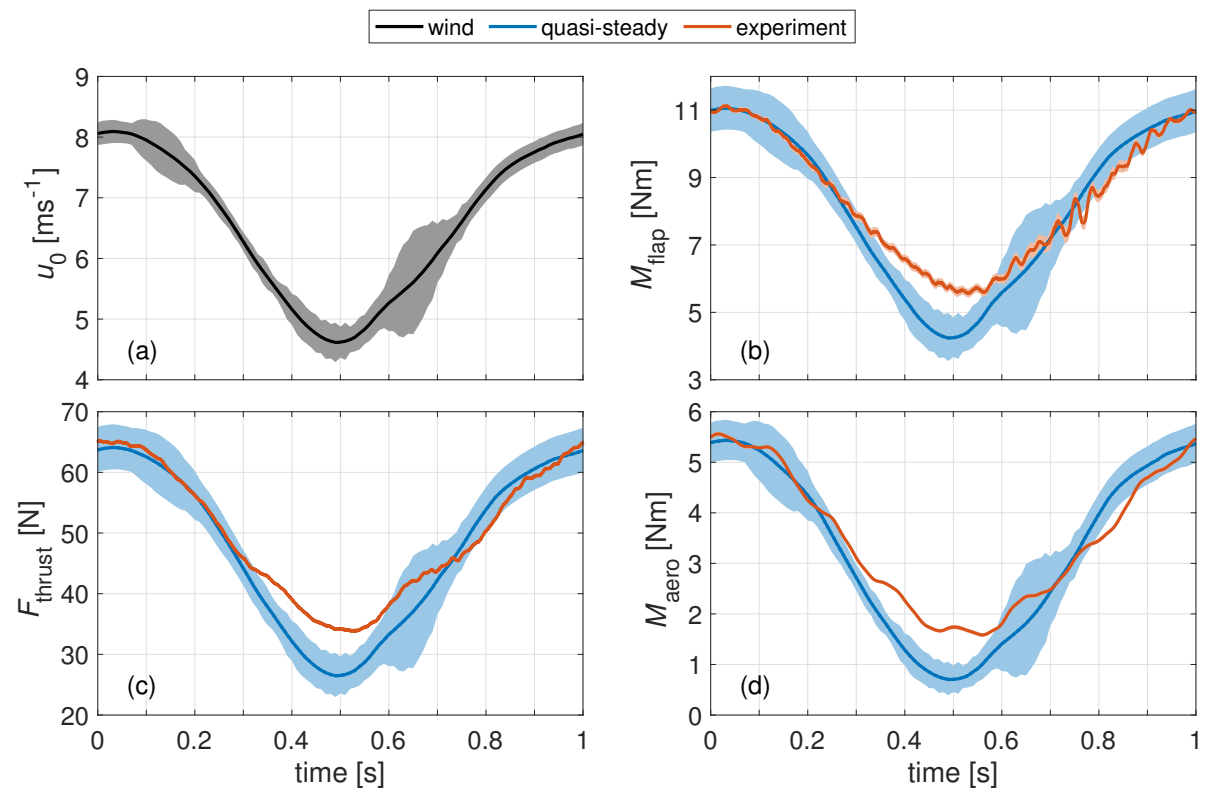

Figure 6. Sine wind field (a) and quasi-steady and dynamic experiment loads for flapwise blade root bending moment (b), thrust (c) and aerodyamic torque (d). The wind uncertainty shows the degree of uniformity. The uncertainty for the quasi-steady loads accounts for this non-uniformity and the uncertainty in the aerodynamic characterisation. The dynamic loads are shown along the $95 \%$ CI.

uncertainty for the wind and $M_{\text {aero }}$ is shown as for the sine gust. The induced velocity is estimated based on the quasisteady thrust coefficient via the momentum balance $\left(C_{\mathrm{T}}=4 a(a-1)\right)$. The time constant was chosen to be half of the typical dynamic inflow value $\left(\tau=\frac{1}{2} \tau_{\text {typ }}=\frac{1}{2} \frac{R}{u_{0, \text { mean }}}=0.07 \mathrm{~s}\right.$, see Schepers and Snel, 1995). With the difference in induced velocity $\Delta u_{\text {ind }}(t)=u_{\text {ind }}(t)-u_{\text {ind }}(t-\tau)$ the difference quotient is $\frac{\Delta u_{\text {ind }}}{\tau}$. A high amount shows a fast change in induced velocity and thus indicates instances where dynamic inflow effects are to be expected based on current engineering models. Additionally, two zoomed-in views of interest, based on relevant differences between steady and dynamic $M_{\text {aero }}$, are shown.

The comparison of the quasi-steady and dynamic $M_{\text {aero }}$ shows a good fit, with only two instances, where the dynamic values are outside of the quasi-steady range. The first instance is shown in zoom 1. The wind velocity (Fig. 7 a1) decreases quickly from $t=8 \mathrm{~s}$ to $t=8.4 \mathrm{~s}$ and the dynamic torque (c1) shows a less pronounced response, similar to the negative gust for the sine variation. The difference quotient of the induced velocity (b1) shows the absolute minimum at $t=8.3 \mathrm{~s}$, coinciding with the maximum difference between experiment and quasi-steady case.

The second instance is around $t=17 \mathrm{~s}$. There is a fast increase in wind velocity (Fig. $7 \mathrm{a} 2$ ) and the dynamic $M_{\text {aero }}$ (c2) does not increase as fast as for the quasi-steady case, thus leading to a less pronounced load peak for the dynamic case. The difference quotient of the induced velocity (b2), in contrast to (b1), does not show an extreme value here. In general there is a slightly reduced variation of $M_{a e r o}$ of the dynamic case in comparison to the steady case, especially at the lower tipping points. Apart from the introduced two instances these differences are all within the uncertainty range. For the two described instances no extrapolated values were needed for the quasi-steady $M_{a e r o}$ signal. 

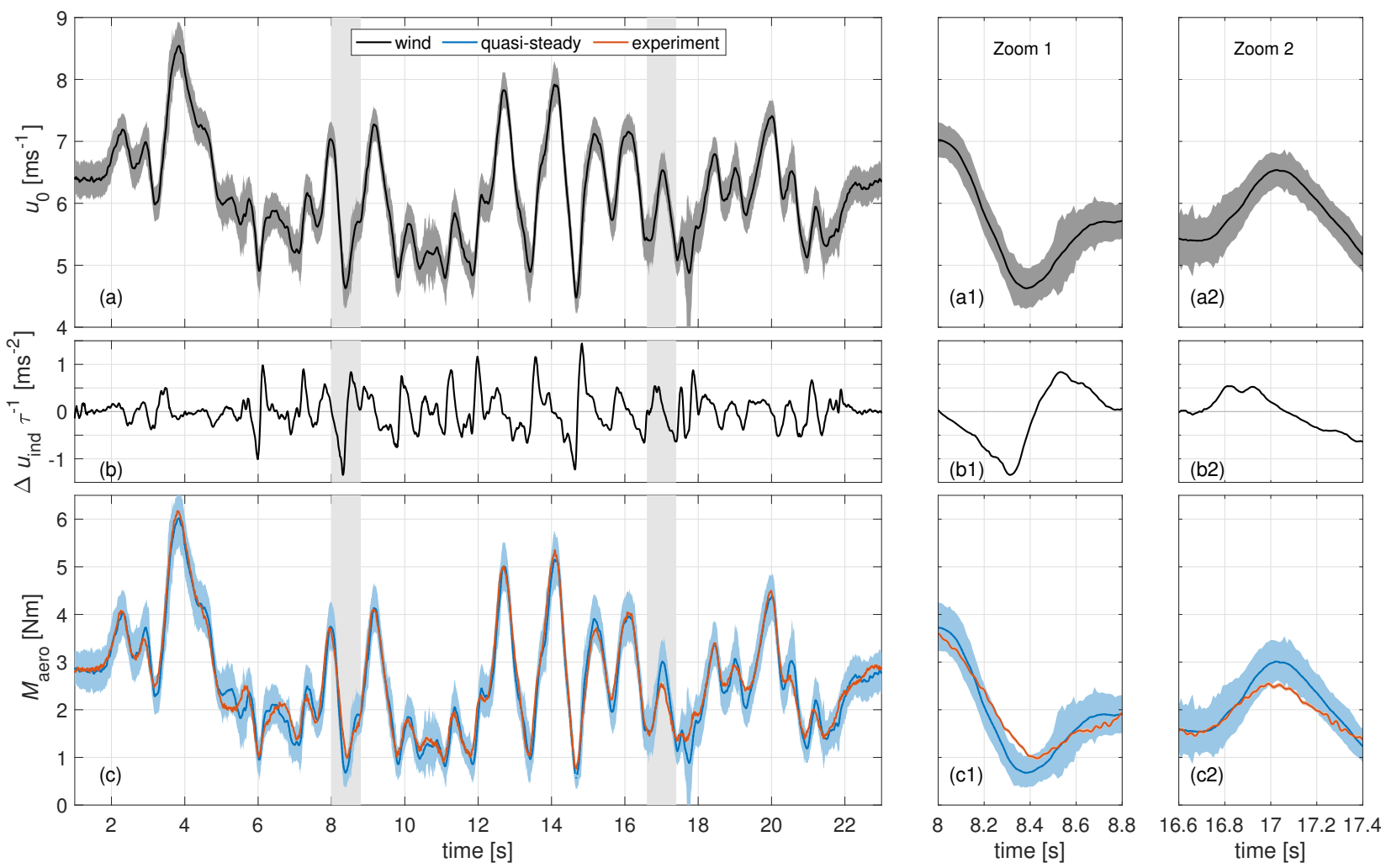

Figure 7. Stochastic wind variation (a), difference quotient of velocity with $\tau$ to obtain the instances of largest relevant velocity change (b) and flapwise blade root bending moment (c) for steady and dynamic case. Furthermore, two zoomed in views (marked in grey in the whole time series) of situations of interest of the same data series are presented (a1,a2,b1,b2,c1,c2).

\subsection{Radius resolved measurement}

For the sine wind field, quasi-steady and dynamic $u_{\text {ax }}$ and $u_{\text {ind }}$ are directly compared for three radii. In the next step all considered radii are used to reconstruct the rotor thrust based on the flow measurement (see Sect. 2.4). This way, the radial measurements are combined to a global signal, reducing uncertainty and noise.

\section{Axial and induced velocity}

In Fig. 8 a, b and c, the axial velocity $u_{\mathrm{ax}}$ for three radii for the dynamic and quasi-steady cases for the sine wind field are shown. For the dynamic case the raw data samples are additionally plotted. The $95 \% \mathrm{CI}$ is given for both signals. For the quasi-steady case this is again altered by the mean wind velocity in the rotor plane at single instances. The dotted line for the quasi-steady case indicates the extrapolated range of the characterisation.

The steady and dynamic axial velocity shows similar behaviour for the three chosen radii. However, differences are evident at the lower tipping points. For all three radii the dynamic case shows higher $u_{\mathrm{ax}}$ values here. At all radii but especially $0.4 R$ 
and $0.6 R$, a plateau can be seen in the dynamic case around $t=0.7 \mathrm{~s}$. This effect is also indicated in the uncertainty band of the quasi-steady case but not in the global mean $u_{0,0.7}$, mean nor the dynamic load measurements. This indicates a local flow pattern that is smoothed out globally.

In Fig. $8 \mathrm{~d}$, e and f, the induced velocity $u_{\text {ind }}=u_{0}-u_{\text {ax }}$ for the steady and dynamic case are plotted for the three radii. The reference velocity for the dynamic case is the corresponding local stand-still measurements $\left(u_{0, \text { local }}\right)$ at the respective measurement position.

The quasi-steady values show a dip by $0.4 \mathrm{~ms}^{-1}$ between $t=0.3 \mathrm{~s}$ and $t=0.5 \mathrm{~s}$ for the radii $0.4 R$ and $0.6 R$. For $0.8 R$ the induced velocity is nearly constant for the whole sine wind variation.

In general the dynamic $u_{\text {ind }}$ has a high level of signal noise. This can be related on the one hand to the combination of small values for $u_{\text {ind }}$ and the noise in the signals $u_{0, \text { local }}$ and $u_{\text {ax }}$. On the other hand $u_{0, \text { local }}$ and $u_{\text {ax }}$ consider the same position, however the effect of the widening stream-tube around the rotor is not considered leading to some possible mismatches in $u_{0, \text { local }}$. Still the comparison gives valuable insights in the phenomenon.

At $0.8 R$ the dynamic $u_{\text {ind }}$ does indicate a steady value, however with higher noise level. For the radius at $0.6 R$ steady and dynamic $u_{\text {ind }}$ start at the same level, whereas the dynamic $u_{\text {ind }}$ increases from $t=0.1 \mathrm{~s}$ to $t=0.2 \mathrm{~s}$ and the quasi-steady signal stays levelled. In the further course the dynamic signal decreases quicker and further from $t=0.3 \mathrm{~s}$ to $t=0.5 \mathrm{~s}$ below the quasi-steady value. From there, the dynamic $u_{i n d}$ does increase to the steady value and again below it around $t=0.7 \mathrm{~s}$, before reaching the steady level again at the end of the sine wind variation. The $0.4 R$ case is similar, with the exception, that the dynamic signal already starts at a higher value than the quasi-steady one. The differences between steady and dynamic $u_{\text {ind }}$ are indications, as the uncertainty range of the quasi-steady case and experiment overlay. However, for some instances, e.g. for $0.4 R$ at $t=0 \mathrm{~s}$ to $t=0.2 \mathrm{~s}$, around $t=0.55 \mathrm{~s}$ and $t=1 \mathrm{~s}$, as well as for $0.6 R$ around $t=0.2 \mathrm{~s}$ and $t=0.75 \mathrm{~s}$ there are more clear indications, as the experimental mean values are outside the uncertainty range of the quasi-steady case.

\section{Reconstructed load}

In Fig. 9, the thrust $F_{\text {thrust,recon, }}$, reconstructed from the measured axial and tangential velocities by Blade Element Theory (see Sect. 2.4), is presented. This reconstructed thrust signal essentially is a spanwise weighted representation of all the axial velocity measurements in one signal, which also can be directly compared to the strain gauge measurement and thus makes both measurements directly comparable. The steady case is based on the spatial mean wind field, as shown in Fig. 6, for which the uncertainty band always encloses $u_{0,0 \mathrm{D}}$, mean. The qualitatively same effect between quasi-steady and dynamic experiment and also the general steady values as for the direct load measurements in Fig. 6 can be seen. The load levels at the top tipping point between dynamic and quasi-steady case are similar. At the bottom tipping point the dynamic case suggests a higher load, leading to lower load amplitude for the dynamic experiment. Due to the uncertainty range of the cases the effect is just clear around $t=0.45 \mathrm{~s}$. The difference at $t=0.7 \mathrm{~s}$ can be linked to the plateau in the axial velocity, that partially is also indicated by the high uncertainty in the quasi-steady case just before that instance. In comparison to the local dynamic measurements shown in Fig. 8 a, b this plateau is smoothened, supporting the assumption of this plateau being a local phenomenon. 
https://doi.org/10.5194/wes-2022-2

Preprint. Discussion started: 18 January 2022

(c) Author(s) 2022. CC BY 4.0 License.

(c) (i)
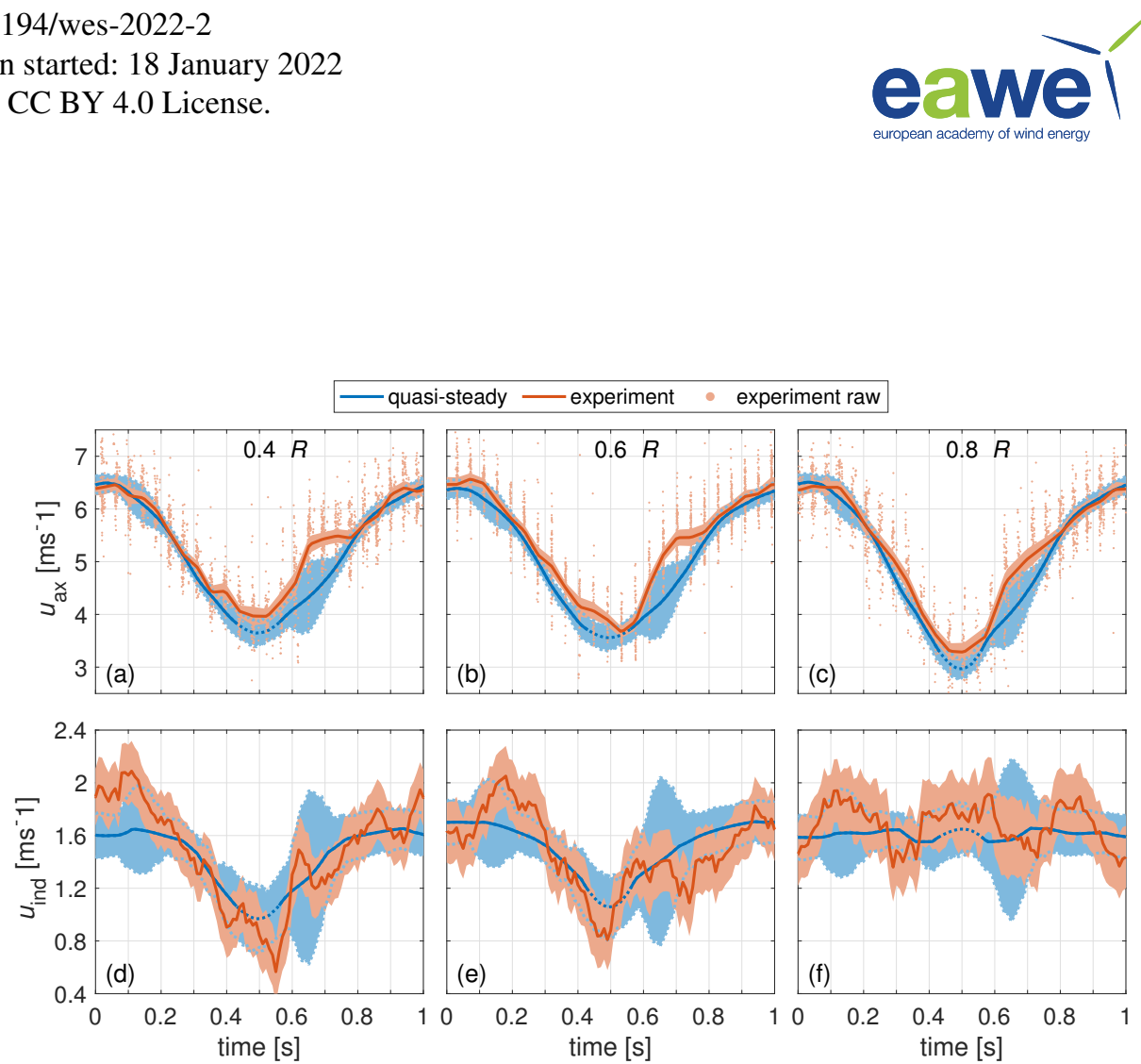

Figure 8. Steady and dynamic axial velocity and induced velocity for sine wind field at radii $0.4 R$ (a, d), $0.6 R$ (b, e) and $0.8 R$ (c, f). $95 \%$ CI are given and errors for quasi-steady case and the induced velocity of the dynamic case were quadratically added.

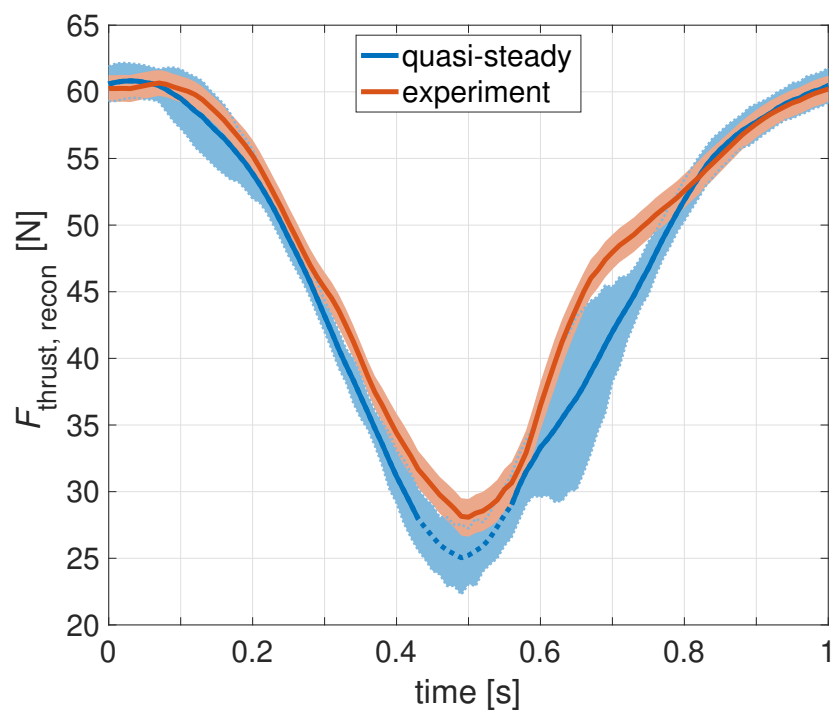

Figure 9. Thrust force reconstructed for the quasi-steady and dynamic experiment from $u_{\mathrm{ax}}$ and $u_{\mathrm{ta}}$ for the sine wind variation. Quasi-steady $u_{\mathrm{ta}}$ is used for both cases due to the low impact. Errorbars are based on the quadratically added $95 \%$ confidence intervals. Dotted values indicate the extrapolated characterisation. 


\subsection{Sine gust in BEM and FVWM}
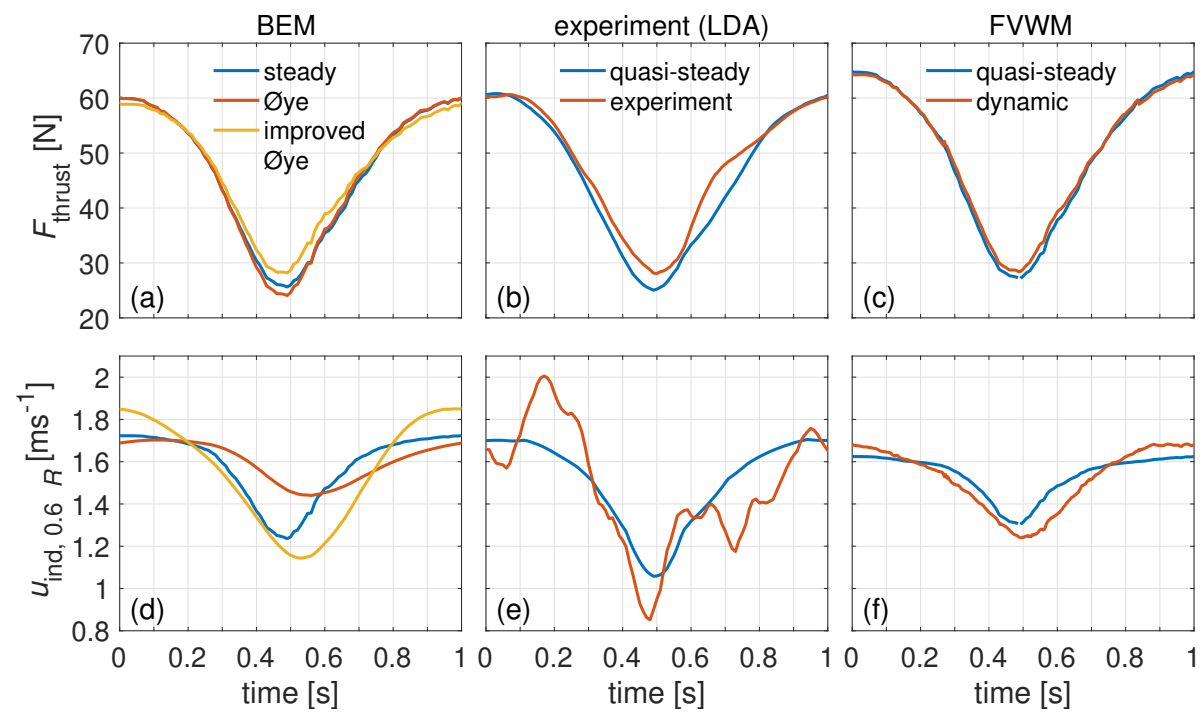

Figure 10. Steady and dynamic thrust (a) and induced velocity (d) at $0.6 \mathrm{R}$ for the sine gust for BEM simulation with the original and improved Øye dynamic inflow model and correspondingly for for the experiment (b,e) and the FVWM simulation (c,f).

In Fig. 10 a $F_{\text {thrust }}$ is shown for the sine wind field in BEM for steady operation without an engineering model and for dynamic operation with the Øye and the improved Øye dynamic inflow model (see Sect. 2.7). A clear difference can be seen between the Øye and the improved Øye model. In relation to the steady case, the Øye model leads to an increase in the load amplitude and the improved Øye model to a decrease. The difference is seen mainly at the lower tipping point.

In Fig. $10 \mathrm{~b}$ and c, $F_{\text {thrust }}$ is shown for the quasi-steady and experimental/dynamic case for the experiment (reconstructed from the LDA measurements; reproduced from Fig. 9) and the FVWM simulation, respectively. In comparison to the steady BEM simulation, quasi-steady values are similar for the experiment and overall slightly higher (about $10 \%$ ) for the FVWM simulation. These differences are negligible here, as the analysis is based on the comparison of different cases of the same simulation environment, respectively experiment.

For experiment and FVWM, the behaviour of the dynamic case shows a reduced load amplitude in relation to the respective quasi-steady case, with the main difference at the lower tipping point at $t=0.5 \mathrm{~s}$. The dynamic inflow effect modelled by the improved Øye implementation for BEM performs similar to the experiment, whereas the effect is less prominent but qualitatively similar in the FVWM simulation. In contrast to the experiment and FVWM, the original Øye model suggests the dynamic effect to increase the load amplitude.

In Fig. $10 \mathrm{~d}$ the induced velocity $u_{\text {ind }}$ at the radius of $0.6 R$ is presented for the steady and dynamic BEM simulation, once with the original and once the improved Øye dynamic inflow model. As by design, the course of the dynamic Øye case is the 
filtered steady signal, reducing the amplitude of $u_{\text {ind }}$ compared to the steady case. In contrast, the improved Øye model leads to a higher amplitude in $u_{\text {ind }}$ compared to the steady case.

In Fig. $10 \mathrm{e}$ and $\mathrm{f}$ the different quasi-steady and dynamic cases of induced velocity $u_{\text {ind }}$ at the radius of $0.6 R$ are presented for the experiment (smoothed; reproduced from Fig. 8 e) and FVWM simulation, respectively. The quasi-steady cases of experiment and FVWM have a similar course as for the BEM simulation, with similar $u_{\text {ind }}$ values at $t=0 \mathrm{~s}$ and some differences at the lower tipping point at $t=0.5 \mathrm{~s}$. As for the thrust these differences are of secondary relevance in this analysis.

The dynamic $u_{\text {ind }}$ of the FVWM simulation shows a very similar course to the improved Øye dynamic inflow model, compared to the respective (quasi-) steady case. This leads to an increased amplitude of the dynamic $u_{\text {ind }}$ compared to the quasi steady case, with slightly higher values at the high tipping point of $F_{\text {thrust }}$ at $t=1 \mathrm{~s}$ and lower values at the lower tipping point at $t=0.5 \mathrm{~s}$. The course of the experimental $u_{\text {ind }}$, compared to the respective quasi-steady case, is less explicit due to the signal noise (see Sect. 3.2). However, the global comparison still shows similarity to the FVWM and improved Øye, leading to an increase in the experimental signal amplitude with lower values at $t=0.5 \mathrm{~s}$.

\section{Discussion}

\subsection{Turbine loads}

The comparison of the steady and dynamic loads of the sine wind variation (see Fig. 6) shows a clear unsteady aerodynamic effect with a reduction in load and rotor torque amplitude for the dynamic case. The main difference is seen around the lower wind tipping point with high TSR and thus also high $C_{\mathrm{T}}$.

The observed dynamic difference has a duration of about $0.3 \mathrm{~s}$, being twice the typical time constant for dynamic inflow phenomena $\left(\tau_{t y p}=\frac{R}{u_{0, \text { mean }}}=0.14 \mathrm{~s}\right)$. In contrast, time constants for unsteady aerodynamic effects on the profile level, like dynamic stall and the Theodorsen effect, range from $1 \mathrm{~ms}$ to $10 \mathrm{~ms}$ here, estimated by the ratio of chord length to relative wind velocity. The exceeding of the stall level at the root (up to $0.25 R$ ) at the high wind velocity tipping point further does not coincide with the phase of interest of the sine gust. We thus rule out a relevant contribution of unsteady profile aerodynamics on the observed effect.

For the stochastic wind variation (see Fig. 7) the same reduction in load amplitude as for the sine gust is seen. The difference quotient in mean rotor induced velocity $\left(\frac{\Delta u_{\text {ind }}}{\tau}\right)$ indicated this reduced response during a fast negative gust.

For the observed reduced load peak due to a positive gust the $\frac{\Delta u_{\text {ind }}}{\tau}$ does not give a clear indication. Similar and higher values are seen for other instances without a clear effect in $M_{\text {aero. }}$. In contrast to other positive gust peaks, the wind tipping point at $t=17 \mathrm{~s}$ is at a lower wind speed of $6.5 \mathrm{~ms}^{-1}$ corresponding to a $C_{\mathrm{T}}$ value of 0.75 in contrast to 0.65 for the highest wind peaks and 0.83 for the lowest wind tipping points. Load variations at high $C_{\mathrm{T}}$ lead to more prominent dynamic inflow effects for the classic case of pitch steps. 


\subsection{Axial and induced velocities in experiment}

The described differences between the steady and dynamic axial velocity (see Fig. 8) at the lower wind tipping point for the sine gust correspond to the effect seen in the load measurements. The lower drop of the axial velocity for the dynamic case at the lower load tipping point corresponds to a smaller reduction of the angle of attack at the respective radius and thus lower fluctuations in both local lift forces and integral load. This lower drop in axial velocity translates to a higher drop in induced velocity.

For $0.8 R$, the steady case experiences a nearly constant induced velocity. Also the dynamic case does not show strong deviations from that level. The levelled behaviour of the steady and dynamic case at this radius is in line with current dynamic inflow modelling, that only reacts on changes in induced velocity. Amplification of the induced velocity is seen for the inner two radii for the dynamic case in comparison to the steady case for the decrease in induced velocity up to the lower tipping point.

The reconstructed steady thrust based on these axial velocity measurements (see Sect. 2.4) shows a good match to the one based on the strain gauge measurement. The slight differences in the absolute levels at the tipping points (lower $-1 \%$; upper $-5 \%$ ) can be attributed to a wide range of influencing parameters including airfoil polars, tip loss model and low numbers of radii where the inductions from the experiment are available. For the direct comparison of steady and dynamic case these influences cancel out as the same model is used.

For the reconstructed thrust qualitatively the same effect as for the direct load measurement at the lower tipping point is seen, leading to a lower load amplitude for the dynamic case. The difference at the lower tipping point between steady and dynamic case here is smaller with $8 \%$ compared to $20 \%$ for the strain gauge measured load, each normalised by the respective quasi-steady maximum to minimum load difference. Considering the $95 \% \mathrm{CI}$, these differences range from $-2 \%$ to $20 \%$ for the LDA reconstructed thrust and from $10 \%$ to $29 \%$ for the strain gauge measured load and do show some overlap.

For the steady and dynamic axial velocity, induced velocity and reconstructed thrust from the rotor flow, a consistent picture to the independent load measurement is given. Therefore, despite the noticeable uncertainty range of these measurements and derived flow quantities this data gives a strong indication of the dynamic inflow effect due to gusts directly in the flow.

\subsection{Comparison to simulations}

The Øye dynamic inflow model was experimentally validated several times, showing accurate predictions for pitch steps, e.g. for integral turbine loads in Snel and Schepers (1994), for the flow field transients in the wake by Yu et al. (2016) and for axial induction transients by Berger et al. (2020). For the investigated sine gust, an increase in dynamic load amplitude is modelled (see Fig. 10 a). In contrast to the BEM simulation with the Øye dynamic inflow model, the experiment and FVWM simulation (see Fig. $10 \mathrm{~b}$ and c) suggest a decrease in dynamic load amplitude.

The increase in dynamic load within the Øye dynamic inflow model is due to the filtering of the induced velocity. Approaching the lower load tipping point in the sine gust the lower drop in induced velocity is equivalent to a higher drop of the dynamic axial velocity. This leads to a higher drop of the angle of attack and thus lower load. This general trend of an increase in load 
amplitude is therefore present for all engineering models that are based on solely filtering the induced velocities (see Schepers and Snel, 1995 for Øye and ECN model, Yu et al., 2019 and Madsen et al., 2020).

The improved Øye dynamic inflow model (see Sect. 2.7) shows the same trend of a decrease in load amplitude as the experiment and the FVWM simulation. Quantitatively the difference between steady and dynamic load at the lower wind tipping point is at $7 \%$, close to the difference in thrust force based on the reconstructed thrust at $8 \%$. The very slightly lower dynamic load at the higher wind tipping point is seen for both the improved Øye model and the FVWM simulations.

The general trend of the induced wind velocity in the improved Øye is similar to the FVWM simulation, however the amplification of the dynamic signal is more pronounced. In comparison, the experiment also indicates a more pronounced amplification of the induced velocity than the FVWM simulations.

This lower amplification in induced velocity of FVWM is in line with the less prominent dynamic load reduction of the FVWM compared to the experiment and the BEM simulation with the improved Øye dynamic inflow model. Together, the FVWM simulations and the experiment give a first validation of the analytically motivated improvements to the Øye dynamic inflow model.

As expected, the dynamic inflow effect due to gusts is caught by the FVWM modelling approach. The less pronounced effect on the loads is suspected to be connected to the non perfect wake convection method, that was observed in a pitch step comparison with the same FVWM model in Berger et al. (2020). Well-tuned FVWM simulations however are expected to be a perfect basis for the development, tuning and validation of dynamic inflow models for gusts.

Given a wider experimental and numerical data basis the improved implementation of the Øye model can be further tuned by changing the time constants $\tau_{\text {slow }}$ and $\tau_{\text {fast }}$ and their weighting $k$. In this way however the performance for pitch steps is changed too, therefore one has to be increased and the other decreased and the ratio $k$ used to keep a comparable performance to the original model. A further tuning possibility that does not impact the pitch performance is to apply the filter not to the far wake velocity $u_{2}$, but an intermediate wake velocity, e.g. $u_{1.5}=u_{0}(1-1.5 a)$.

\subsection{Normalised comparison to Joule experiment and free field}

510 In contrast to our findings, Snel and Schepers (1994), found no dynamic inflow effect due to gusts in their wind tunnel study with a $1.2 \mathrm{~m}$ diameter model wind turbine and a gust generator for approximating stepwise changes in wind velocity. Starting at an initial wind speed of $5.7 \mathrm{~ms}^{-1}$ they reduced the wind velocity by $0.8 \mathrm{~ms}^{-1}$ within $0.4 \mathrm{~s}$ leading to a nearly linear decrease of induced velocity for the step to lower wind velocity by a maximum amount of $0.3 \mathrm{~ms}^{-1}$, estimated from simulations. Devided with the respective representative dynamic inflow time constant $\tau=\frac{1}{2} \tau_{\mathrm{typ}}=\frac{1}{2} \frac{R}{u_{0, \text { mean }}}$ (see Sect. 3.1), the maximum 515 difference quotient of the induced velocity accounts to $\frac{\Delta u_{\text {ind }}}{\tau}=0.34 \mathrm{~ms}^{-2}$.

For the presented experiment, there are nearly four times higher values at $\frac{\Delta u_{\text {ind }}}{\tau}=1.28 \mathrm{~ms}^{-2}$, with $\Delta u_{\text {ind }}=0.4 \mathrm{~ms}^{-1}$ within $0.2 \mathrm{~s}$ for $0.6 R$ at approximately $t=0.4 \mathrm{~s}$ for the sine variation. Snel and Schepers (1994) already concluded that their change in wind velocity is not sufficiently fast to trigger clear dynamic inflow effects. This nearly four times higher $\frac{\Delta u_{\text {ind }}}{\tau}$ is a plausible explanation why the effect can be seen in the present study. 
Using scaling (see Sect. 2.1) the corresponding gust events for multi MW turbines can be estimated. This would result in a sine gust with a mean wind velocity of $9.0 \mathrm{~ms}^{-1}$ and amplitude of $2.5 \mathrm{~ms}^{-1}$ for the NREL 5MW reference turbine with a gust length of $50 \mathrm{~s}$. This is a realistic value for a gust in the open field.

\section{Conclusions}

We experimentally prooved the dynamic inflow effect due to gusts for wind turbines. We tested if current dynamic inflow engineering models are able to predict the effect and proposed an improvement based on analytical considerations.

Firstly, experiments under reproducible gust conditions and highly resolved measurements of the longitudinal wind field prooved a dynamic inflow effect due to gusts. The effect leads to damped load amplitudes and thus reduced fatigue loads. This was observed most clearly for negative gust cases at high thrust coefficients and attributed to high changes in induced velocity. For positive gusts, the effect was less pronounced and only seen for one high thrust coefficient configuration.

The dynamic inflow effect is also seen in the measurements of axial flow and induced velocity in the rotor plane. The effect leads to an amplification of the induced velocities. The effect is also seen in FVWM simulations for the loads and induced velocity. Widely applied engineering models that filter the induced velocities, like Øye model (Snel and Schepers, 1994) in GH Bladed and OpenFAST, the new DTU model (Madsen et al., 2020) in HAWC2 and ECN model (Snel and Schepers, 1994) in Phatas, cannot adequately catch the dynamic inflow phenomenon due to gusts. They damp the induced velocity due to the filtering, thus leading to higher fatigue loads. As an initial model to tackle the dynamic inflow effect due to gusts we proposed an improvement in the implementation of the Øye model where the filter with the slow time constant acts on the wake flow rather than the induced velocity. This leads to the observed reduced load amplitudes during gusts with an unchanged performance for pitch cases.

Now, that the effect is known further, pinpointed wind tunnel experiments are needed for the development, tuning and validation of dynamic inflow models for gusts. One focus should be to further reduce uncertainties, especially in the inflow. Furthermore, the typical operation of variable speed controlled wind turbine in the free field is more complex than the presented wind tunnel test. Comparisons between FVWM and BEM simulations similar to Perez-Becker et al. (2020) and Boorsma et al. (2020) but without sheared inflow can further shed light on the effect, help to quantify the actual reduction in fatigue loads in realistic turbine operation and serve as a reality near validation method for new dynamic inflow models. These new findings of dynamic inflow due to gusts are a major step to improved dynamic inflow modelling of gusts. The proposed improvement to the Øye dynamic inflow model already provides a possible first generation dynamic inflow model to catch the general effect in BEM simulations. As this effect leads to lower fatigue loading of wind turbines, a proper and validated model opens up new design opportunities. For this aim further coordinated research work is proposed, consisting of wind tunnel experiments and FVWM simulations. 
https://doi.org/10.5194/wes-2022-2

Preprint. Discussion started: 18 January 2022

(C) Author(s) 2022. CC BY 4.0 License.

(c) (1)

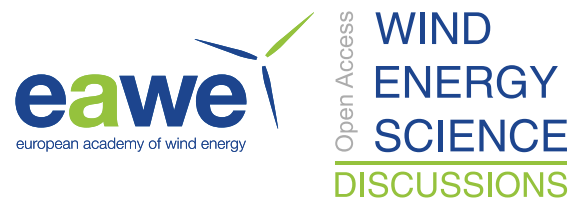

550 Code and data availability. Time series of the measurements will be made available with final publishing. Turbine documentation for simulations is available at https://doi.org/10.5281/zenodo.5552171 (Berger et al., 2021b). QBlade (v0.963) is an open source code that is available online at https://sourceforge.net/projects/qblade (QBlade, 2021).

Author contributions.

FB designed, performed, processed and analysed experiment and simulations, proposed the model improvements and wrote

555 the manuscript. LN developed the protocols for the active grid and assisted in the experiment. DO assisted in the experiment and protocol iterations. MH, GS and MK contributed with several fruitful discussions. MK supervised the work. All authors reviewed the manuscript.

Competing interests. The authors declare that they have no conflict of interest.

Acknowledgements. This work was partially funded by the Ministry for Science and Culture of Lower Saxony through the funding initiative

560 Niedersächsisches Vorab in the project "ventus efficiens" (reference no. ZN3024). We thank Apostolos Langidis for the help in the wind tunnel experiment and Lars Kröger, Lisa Rademacher and Tom Wester for their help with the LDA. 
https://doi.org/10.5194/wes-2022-2

Preprint. Discussion started: 18 January 2022

(C) Author(s) 2022. CC BY 4.0 License.

\section{References}

Berger, F., Kröger, L., Onnen, D., Petrović, V., and Kühn, M.: Scaled wind turbine setup in a turbulent wind tunnel, J. Phys.: Conf. Ser., 1104, 012 026, https://doi.org/10.1088/1742-6596/1104/1/012026, 2018.

Berger, F., Höning, L., Herráez, I., and Kühn, M.: Comparison of a radially resolved dynamic inflow pitch step experiment to mid-fidelity simulations and BEM, J. Phys.: Conf. Ser., 1618, 052 055, https://doi.org/10.1088/1742-6596/1618/5/052055, 2020.

Berger, F., Onnen, D., Schepers, G., and Kühn, M.: Experimental analysis of radially resolved dynamic inflow effects due to pitch steps, Wind Energ. Sci., 6, 1341-1361, https://doi.org/10.5194/wes-6-1341-2021, 2021a.

Berger, F., Onnen, D., Schepers, G., and Kühn, M.: Dataset - Radially resolved dynamic inflow pitch step experiment in wind tunnel, Zenodo [data set], https://doi.org/10.5281/zenodo.5552171, 2021b.

Boorsma, K., Wenz, F., Lindenburg, K., Aman, M., and Kloosterman, M.: Validation and accommodation of vortex wake codes for wind turbine design load calculations, Wind Energ. Sci., 5, 699-719, https://doi.org/10.5194/wes-5-699-2020, 2020.

Buhl, M. L. J.: A New Empirical Relationship between Thrust Coefficient and Induction Factor for the Turbulent Windmill State, Tech. Rep. NREL/TP-500-36834, National Renewable Energy Laboratory, Golden, 2005.

575 Drela, M.: An Analysis and Design System for Low Reynolds Number Airfoils, in: Low Reynolds Number Aerodynamics, vol. 54 of Lecture Notes in Engineering, pp. 1-12, Springer Berlin Heidelberg, https://doi.org/10.1007/978-3-642-84010-4, 1989.

Hansen, M. O. L.: Aerodynamics of Wind Turbines, Earthscan London, 2008.

Herráez, I., Daniele, E., and Schepers, J. G.: Extraction of the wake induction and angle of attack on rotating wind turbine blades from PIV and CFD results, Wind Energ. Sci., 3, 1-9, https://doi.org/10.5194/wes-3-1-2018, 2018.

Jonkman, J., Butterfield, S., Musial, W., and Scott, G.: Definition of a 5-MW Reference Wind Turbine for Offshore System Development, Tech. Rep. NREL/TP-500-38060, National Renewable Energy Laboratory (NREL), Golden, 2009.

Kröger, L., Frederik, J., Van Wingerden, J. W., Peinke, J., and Hölling, M.: Generation of user defined turbulent inflow conditions by an active grid for validation experiments, J. Phys.: Conf. Ser., 1037, 052 002, https://doi.org/10.1088/1742-6596/1037/5/052002, 2018.

Madsen, H. A., Larsen, T. J., Pirrung, G. R., Li, A., and Zahle, F.: Implementation of the blade element momentum model on a polar grid and its aeroelastic load impact, Wind Energ. Sci., 5, 1-27, https://doi.org/10.5194/wes-5-1-2020, 2020.

Marten, D., Lennie, M., Pechlivanoglou, G., Nayeri, C. N., and Paschereit, C. O.: Implementation, Optimization, and Validation of a Nonlinear Lifting Line-Free Vortex Wake Module Within the Wind Turbine Simulation Code qblade, J. Eng. Gas Turb. Power, 138, 072 601, https://doi.org/10.1115/1.4031872, 2016.

Medici, D., Ivanell, S., Dahlberg, J., and Alfredsson, P.: The upstream flow of a wind turbine: blockage effect, Wind Energy, 14, 691-697, https://doi.org/10.1002/we.451, 2011.

Neuhaus, L., Berger, F., Peinke, J., and Hölling, M.: Exploring the capabilities of active grids, Exp. Fluids, 62, 130, https://doi.org/10.1007/s00348-021-03224-5, 2021.

Perez-Becker, S., Papi, F., Saverin, J., Marten, D., Bianchini, A., and Paschereit, C. O.: Is the Blade Element Momentum theory overestimating wind turbine loads? - An aeroelastic comparison between OpenFAST's AeroDyn and QBlade's Lifting-Line Free Vortex Wake method, Wind Energ. Sci., 5, 721-743, https://doi.org/10.5194/wes-5-721-2020, 2020.

Peters, D. A.: How Dynamic Inflow Survives in the Competitive World of Rotorcraft Aerodynamics, J. Am. Helicopter Soc., 54, 11 0011100 115, https://doi.org/10.4050/JAHS.54.011001, 2009. 
https://doi.org/10.5194/wes-2022-2

Preprint. Discussion started: 18 January 2022

(c) Author(s) 2022. CC BY 4.0 License.

(c) (1)

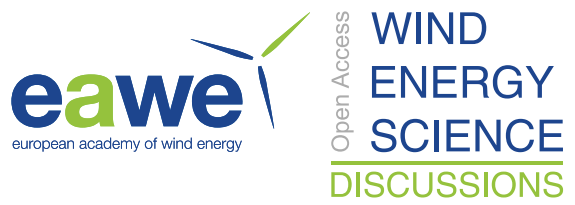

Pirrung, G. R. and Madsen, H. A.: Dynamic inflow effects in measurements and high-fidelity computations, Wind Energ. Sci., 3, 545-551, https://doi.org/10.5194/wes-3-545-2018, 2018.

600 QBlade: available at: https://sourceforge.net/projects/qblade, accessed: September 30. 2021, 2021.

Schepers, J. and Snel, H.: Dynamic inflow: yawed conditions and partial span pitch control, Tech. Rep. ECN-C-95-056, Energy research Center of the Netherlands, Petten, 1995.

Shen, W. Z., Mikkelsen, R., Sørensen, J. N., and Bak, C.: Tip loss corrections for wind turbine computations, Wind Energy, 8, 457-475, https://doi.org/https://doi.org/10.1002/we.153, 2005.

Shirzadeh, K., Hangan, H., Crawford, C., and Hashemi Tari, P.: Investigating the loads and performance of a model horizontal axis wind turbine under reproducible IEC extreme operational conditions, Wind Energ. Sci., 6, 477-489, https://doi.org/10.5194/wes-6-477-2021, 2021.

Snel, H. and Schepers, J.: Joint investigation of dynamic inflow effects and implementation of an engineering method, Tech. Rep. ECN-C94-107, Energy research Center of the Netherlands, Petten, 1994.

610 Snel, H., Houwink, R., Bosschers, J., Piers, W.J.; van Bussel, G., and Bruining, A.: Sectional prediction of 3-D effects for stalled flow on rotating blades and comparison with measurements, Tech. Rep. ECN-RX-93-028, Energy research Center of the Netherlands, Petten, 1993.

Van Garrel, A.: Development of a Wind Turbine Aerodynamics Simulation Module, Tech. Rep. ECN-C-03-079, Energy research Center of the Netherlands, Petten, 2003.

615 Yu, W., Hong, V., Ferreira, C., and Van Kuik, G. A. M.: J. Phys.: Conf. Ser., 753, 022 024, https://doi.org/10.1088/1742-6596/753/2/022024, 2016.

Yu, W., Tavernier, D., Ferreira, C., van Kuik, G. A., and Schepers, G.: New dynamic-inflow engineering models based on linear and nonlinear actuator disc vortex models, Wind Energy, 22, 1433-1450, https://doi.org/10.1002/we.2380, 2019. 